\title{
Model-Based Security Testing
}

\author{
Ina Schieferdecker \\ Fraunhofer FOKUS \\ Berlin, Germany \\ Freie Universitaet Berlin \\ Berlin, Germany \\ ina.schieferdecker@fokus.fraunhofer.de \\ Juergen Grossmann \\ Fraunhofer FOKUS \\ Berlin, Germany \\ juergen.grossmann@fokus.fraunhofer.de \\ Martin Schneider \\ Fraunhofer FOKUS \\ Berlin, Germany \\ martin.schneider@fokus. fraunhofer.de
}

\begin{abstract}
Security testing aims at validating software system requirements related to security properties like confidentiality, integrity, authentication, authorization, availability, and non-repudiation. Although security testing techniques are available for many years, there has been little approaches that allow for specification of test cases at a higher level of abstraction, for enabling guidance on test identification and specification as well as for automated test generation.

Model-based security testing (MBST) is a relatively new field and especially dedicated to the systematic and efficient specification and documentation of security test objectives, security test cases and test suites, as well as to their automated or semi-automated generation. In particular, the combination of security modelling and test generation approaches is still a challenge in research and of high interest for industrial applications. MBST includes e.g. security functional testing, model-based fuzzing, risk- and threat-oriented testing, and the usage of security test patterns. This paper provides a survey on MBST techniques and the related models as well as samples of new methods and tools that are under development in the European ITEA2-project DIAMONDS.
\end{abstract}

\section{Introduction}

The times of rather static communication in strictly controlled, closed networks for limited purposes are over, while the adoption of the Internet and other communication technologies in almost all domestic, economic and social sectors with new approaches for rather dynamic and open networked environments overwhelmingly progresses. Social networks, networked communities, cloud computing, Web X.0, mashups or business process design are just some of the trends that reflect the tendency towards permanent connections and permanent data collection. Today's networked systems face the challenge of various security threats, which is usually met by various protection systems against attacks from the direct system users. In an interconnected world, software with vulnerabilities presents a threat not only to individuals but also to companies and public organizations, and last but not latest to national and international cooperation. Compared with functional hazards, which tend to be straightforward and accidental, security threats are often intentional and more persistent. Here are some facts that highlight the special nature of security threats:

- Attacks are frequently carried out by well organized groups with a commercial background (spamming, extortion, industrial espionage)

- Multi-stage attacks skilfully combine vulnerabilities on system level and organizational level

A. Petrenko, H. Schlingloff (Eds.): Workshop on Model-Based Testing 2012 (MBT 2012)

EPTCS 80, 2012, pp. 1-12, doi $10.4204 /$ EPTCS.80.1 (c) I. Schieferdecker, J. Grossmann, M. Schneider

This work is licensed under the Creative Commons Attribution License. 
- information security risk analysis does often not hold for the complete life time of a product (context of product usage may change, new vulnerabilities are detected)

Practical security involves a sufficient understanding of risk in order to properly address it, manage it and, with care, to eliminate it. National and international standardization committees provide significant efforts on security evaluation and assessments. It includes classical concepts from security evaluation using common criteria (CCRA), but also European activities from ETSI addressing risk and threat analysis (TVRA).

The main aim of information security methods and techniques is the reduction or elimination of unwanted incidents that may harm the technical infrastructure or, even worth, its environment. The field of information security has been growing and reaches far further than simply using cryptography. The purpose of today's security standards is to specify countermeasures that can protect a system against certain forms of exploitation. Countermeasures depend on an understanding primarily on finding and assessing the vulnerabilities that exist within a system. Testing can be seen as an action to proactively detect such vulnerabilities. The Software Engineering Institute, USA, highlighted in 2009: "The security of a software-intensive system is directly related to the quality of its software." About 90 percent of all software security incidents are caused by attackers who exploit known vulnerabilities. Moreover most known vulnerabilities originate from software faults or design flaws. However, not every fault or design flaw constitutes vulnerability. Still, systematic testing increases the likelihood of identifying faults and vulnerabilities during the design, development or setup time of systems and enables purposeful fixes.

\section{Model-based security testing and security testing}

Software testing is an experimental approach of validating and verifying that a software system meets its functional and extra-functional requirements and works as expected. In this article, testing refers to active, dynamic testing, where the behavior of a system under test (SUT) is checked by applying intrusive tests that stimulate the system and observe and evaluate the system reactions. This is done by applying specification-based and/or code-based test cases that are - based on test hypotheses - directed to find faults in the SUT and by providing test suites, i.e. specifically selected collections of test cases, which provide an argument for the absence of faults.

Software security testing is a special kind of testing with the aim in validating and verifying that a software system meets its security requirements. Two principal approaches can be used: functional security testing and security vulnerability testing [5]. While security functional testing is used to check the functionality, efficiency and availability of the designed and developed security functionalities and/or security systems (e.g. firewalls, authentication and authorization subsystems, access control), security vulnerability (or penetration) testing directly addresses the identification and discovery of yet unknown system vulnerabilities that are introduced by security design flaws or by software defects. Security vulnerability testing uses the simulation of attacks and other kinds of penetration attempts.

The systematic identification and reduction of security-critical software vulnerabilities and of defects will increase the overall dependability of software-based systems and helps providing adequate security levels for open systems and environments. Unfortunately security testing, especially security vulnerability testing, lacks systematic approaches, which enable the efficient and goal-oriented identification, selection and execution of test cases. Risk-based testing [4] is a methodology that makes software risks the guiding factor to solve decision problems in the design, selection and prioritization of test cases. 


\subsection{Related work}

The basic idea of model-based testing (MBT) is that instead of creating test cases manually, selected algorithms are generating them automatically from a (set of) model(s) of the system under test or of its environment. While test automation replaces manual test execution by automated test scripts, modelbased testing replaces manual test designs by automated test generation. Although there are a number of research papers addressing model-based security (see e.g. [2, 9] and model-based testing (see e.g. [1]), there is until today little work on model-based security testing (MBST). Relevant publications in the field of MBST are [3, 10, 11, 16, 21, 23, 12, 19].

Kaksonen et al. [12] from the PROTOS project (1999-2001) discuss and implement an MBST approach using syntax testing as the starting point, and implement the models using Augmented BackusNaur Form (ABNF). The PROTOS approach to model-based testing reads in context-free grammars (defined by BNF, ASN.1 (Abstract Syntax Notation One),or XML (Extended Markup Language)) for critical protocol interfaces and generates the tests by systematically walking through the protocol behaviour. These two approaches are the only ones commercially available [19].

Jurjens and Wimmel [11,23] address the problem of generating test sequences from abstract system specifications in order to detect possible vulnerabilities in security-critical systems. Both papers assume that the system specification, from which tests are generated, is formally defined in the language Focus - a mathematical framework for the specification, refinement, and verification of distributed, reactive systems. The paper [11] focuses on testing of firewalls whereas [23] focuses on transaction systems. In [10], Jurjens extends [8] by considering system specification written in the language UMLsec - a security profile for the Unified Modelling Language. In [3], Blackburn et. al. summarizes the results of applying a model-based approach to automate functional security testing. The approach involves developing models of security requirements as the basis for automatic test vector and test driver generation. In particular, security requirements are written in the so-called SCRtool, are transformed into test specifications which in turn are transformed into test vectors and test drivers. The approach is targeted towards Java applications and database servers.

Mouelhi et al. [16] propose a model-driven approach to specifying, deploying and testing accesscontrol policies in Java applications. The approach has four main steps. The first step is to build a platform-independent access-control model for the application. In the second step, the model is transformed into so-called platform specific policy decisions points (PDPs). In step three, the PDP is integrated into the functional code of the application by aspect oriented programming techniques. Finally, in step four, the resulting integrated application is tested against tests that are generated from the platform independent access-control model. Another approach covering specification, deployment, testing and monitoring of security policies has been proposed in the Politess project (Grenoble INP, IT, Smartesting) [DFGMR06, MBC08, LR07]. In [21], Wang et. al. presents a threat driven approach to MBST. In this approach, UML sequence diagrams to specify threat a model, i.e., event sequences that should not occur during the system execution. The threat model is then used as a basis for code instrumentation. Finally, the instrumented code is recompiled and executed using randomly generated test cases. If an execution trace matches a trace described by the threat model, security violations are reported and actions should be taken to mitigate the threat in the system.

\subsection{Models in model-based security testing}

In order to test security properties information from different sources are needed and need to be systematically related to each other to support tracing and proper usage of the information. In addition to 
the functional system specification and to system architecture information, information on known or potential vulnerabilities, potential attacks and their occurrence probabilities can give guidance on what to test and how to test. In addition, probabilities and estimations on the severity of potential attacks can be summed up to form a risk analysis that points at the threats that are to be considered. Such a risk analysis provides guidance for test ordering and test prioritization and supports test management by indicating the need for test recommended test resources.

Hence, model-based security testing needs to be based on different types of models in order to cover the different perspectives used in securing a system. In the following we provide three different model categories that each represent a perspective on its own and may serve as input models for test generation.

\subsubsection{Architectural and functional models}

Architectural and functional models of the SUT are concerned with system requirements regarding the general behaviour and setup of a software-based system. The main perspective of these models are the structure and properties of the system under test. The models exist on different level of abstraction and in different granularity. Often they show additions that allow to focus on specific system properties like robustness properties (e.g. failure states) or performance properties (e.g. durations or throughputs). Regarding security testing, we are principally interested in locating critical system functionality with respect to the overall software architecture and in identifying security-critical interfaces, which might be an entry point for an adversary. Related to security-critical interfaces, interaction models or protocol models (involving data models or behavioural models) are of high interest for security testing. In addition functional security measures (such as authentication or access control means) can be specified within functional models and be tested by use of functional testing approaches.

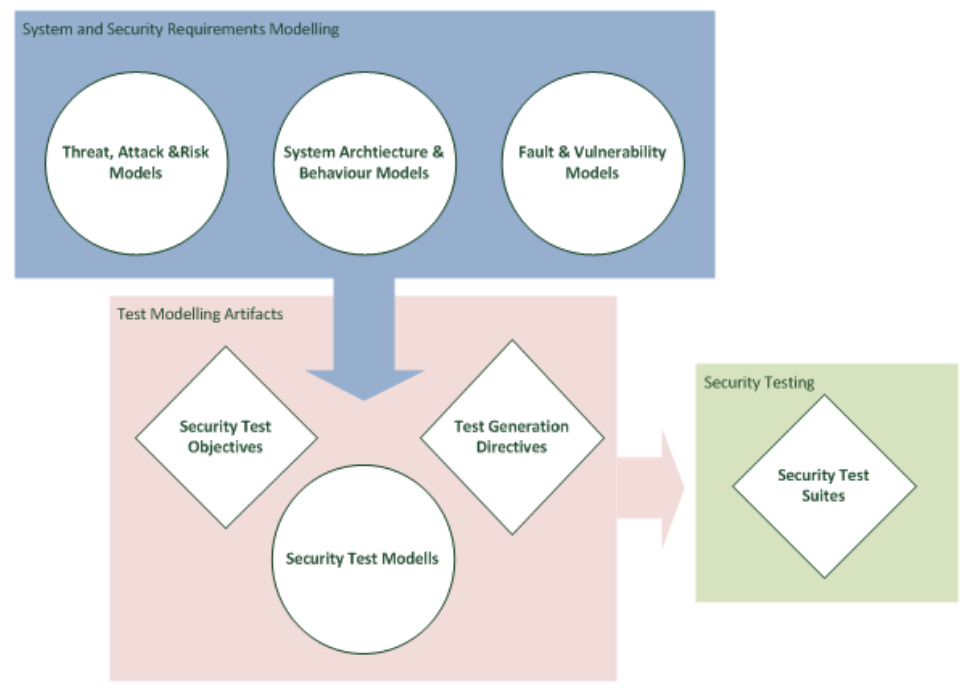

Figure 1: Modelling Artifacts in MBST

\subsubsection{Threat, fault and risk models}

While architectural and functional models typically describe the expected system configuration and behaviour, risk modelling techniques like the CORAS risk modelling approach [13, 7] focus on what can 
go wrong. CORAS provides means to mode risks, threats or faults and enables the identification of multiple risk factors, describe their relationships and relate them to occurrence probabilities and potential impacts.

Besides CORAS, further approaches for fault and attack modelling exist. Well-known are the fault tree analysis (FTA [20]) and the cause-consequence analysis (CCA [17]). FTA considers high-level faults and decomposes them top-down to basic events, which can be identified and tested for in the system.

A variant of fault trees are so called attack trees [14]. Attack trees are directly related to security risks. They start from a high-level attack scenario and decompose them to concrete basic interactions with the system.

ETA (event tree analysis [18]) works bottom-up. It starts with the identification of unwanted system events and analyses the consequences in case of an occurring unwanted event.

A CCA (cause-consequence analysis [6]) - a combination of the FTA and ETA concepts - can be used as well. That analysis starts with a thread. The causes (top-town) and the consequences (bottom-up) are analyzed simultaneously.

\subsubsection{Weakness and vulnerabilities models}

While threat, fault and risk models concentrate on causes and consequences of system failures, weaknesses or vulnerabilities, a weakness or vulnerability model describes the weakness or vulnerability by itself. The information needed to develop such models are normally given by databases like the National Vulnerability Database (NVD) or the Common Vulnerabilities and Exposures (CVE) database. These databases collect known vulnerabilities and provide the information to developers, testers and security experts, so that they can systematically check their products for known vulnerabilities. One of the challenges yet not sufficiently solved is how these vulnerabilities can be integrated in system models, so that they can be used for test generation.

One possible solution is based on the idea of mutation testing [22]. Typically, mutation testing is used to qualify test suites by running tests against a mutation of the system under test. The quality of the test suite is stated with respect to the number of mutants being detected by the test suite. For security testing, models of the system under test are mutated in a way that the mutants represent weaknesses or known vulnerabilities. These weakness or vulnerability models can then be used for test generation by various MBT approaches. The generated tests are used to check whether the system under test is weak or vulnerable with respect to the weaknesses and vulnerabilities in the model.

\subsection{Activities in model-based security testing}

Security testing like any other testing follows a series of activities and uses artifacts that aim to systematically plan, design, specify, realize, and execute tests, and to evaluate the test results and, if needed, to readjust the planning etc.

MBST shows slight differences in these activities, but follows the main sequence of activities. A main task is to provide system properties under consideration concrete test cases (data and behaviour) that represent the stimuli to the system under test and the evaluation of the system reactions by test oracles. The following discusses how to generate security tests with a model-based approach.

- Identify security test objectives and methods: The test objectives define the overall goals of testing and relate the goals to testing methods that allow to accomplish the objectives. For model-based testing the modelling techniques and test generation strategies need to be planned. Especially 
threat, fault, and risk models are to be considered to guide or strengthen the test identification with respect to the identified risks, threats, faults, and their consequences.

- Design a functional test model: The test model reflects either the expected functional scenarios of the SUT (system perspective) or the scenarios of the SUT usage (system perspective). Standard modelling languages such as UML can be used to formalize the points of control and observation of the SUT, the dynamic behaviour when interacting with the system, the entities associated with the test in various test configurations, and the test data applied to the system. The test models need to be precise and complete enough to allow automated derivation of executable tests from these models. However, security testing focuses either on testing the correctness of security functions or on testing the robustness against a dedicated misuse of the system. Thus, functional test models used for security testing describe not only the typical environment or usage of a system, but also adversary environments or atypical usages like attacks and hacking attempts.

- Determine test generation criteria: Usually, there is an infinite number of possible tests that can be generated from a model, so that test designers choose test generation or selection criteria to limit the number of (generated) tests to a finite number by e.g. selecting highest-priority tests, or to ensure specific coverage of system structures, behaviours, or alike. For security testing, approaches based on structural model coverage, i.e. determining the coverage of model elements by generated tests, is not sufficient. Hence, fuzz test approaches are used that follow other kind of coverage criteria and lead to different, but also larger number of generated test data or test behaviour. However, approved test generation criteria like the coverage of security functional requirements or the weighting of security functional requirements with risk values are applicable as well.

- Generate the tests: The test generation is in MBT typically a fully automated process to derive the test cases from a given test model as determined by the chosen test generation criteria. This is also true for MBST. The generated test cases are sequences of high-level events or actions to or from the SUT, with input parameters and expected output parameters and return values for each event or action. If needed, the generated tests are further refined to a more concrete level or adapted to the SUT to support their automated execution.

- Assess the test results: During test result evaluation and test assessment, the quality of the SUT can be rated with respect to the test results as well as the quality of tests can be rated with respect to their fault and vulnerability revealing capabilities. While for functional MBT, measurements and metrics for system and test quality exist this is still a research challenge for MBST. Furthermore, the test results need to be analyzed if changes to the system requirements, the system design, the risk analysis or to the test process itself are needed. In these cases, required iterations are to be started.

\subsection{Testing Approaches in DIAMONDS}

The project DIAMONDS (Development and Industrial Application of Multi-Domain Security Testing Technologies) develops under the direction of Fraunhofer FOKUS, Berlin, efficient and automated security test methods for security-critical, networked systems in various industrial domains such as industrial automation, banking and telecommunications. DIAMONDS develops methods to design objective, transparent, repeatable, and automated security tests that focus on system specifications and related risks. The project goals include the definition of security fault and vulnerability modelling techniques, the definition of a security test pattern catalogue, the development of MBST techniques, and the definition of 
a MBST methodology. DIAMONDS examines vulnerabilities of networked systems in the considered domains in order to derive common principles, methods and means that enable effective security testing of industrial importance. In reflection of the case studies results, the DIAMONDS security testing methodology will be evaluated and optimized. The project results are made available to interested parties and also through contributions to the standardization at ETSI and to other standardization bodies.

A special focus is given in DIAMONDS to (1) risk-based MBST and to (2) model-based fuzz testing.

\subsubsection{Risk-based security testing}

Risk-based testing can be generally introduced with two different goals in mind. On the one hand side risk-based testing approaches can help to optimize the overall test process. The results of the risk analysis, i.e. the results of threat and vulnerability analysis, are used to guide the test identification and may complement requirements engineering results with systematic information concerning threats and vulnerabilities of a system. On the other hand side, attack simulation is to find deviations of the SUT to its specification that leads to vulnerabilities because invalid inputs are not rejected but processed by the SUT instead. Such deviations may lead to undefined states of the SUT and can be exploited by an attacker, for example to successfully perform a denial-of-service.

A comprehensive risk assessment additionally introduces the notion of risk values, that is the estimation of probabilities and consequences for certain threat scenarios. These risk values can be additionally used to weight threat scenarios and thus help identifying which threat scenarios are more relevant and thus identifying the threat scenarios that are the ones that need to be treated and tested more carefully.

Furthermore, risk-based testing approaches can help to optimize the risk analysis and the risk assessment itself. Risk analysis and risk assessment, similar to other development activities in early project phases, are mainly based on assumptions on the system itself. Testing is one of the most relevant means to do real experiments with a system and thus enables to gain empirical evidence on the existence of vulnerabilities, the applicability and consequences of threat scenarios and the quality of countermeasures. Thus, risk-based testing results can be used as a form of evidence for the assumptions that have been made during the risk evaluation and risk assessment.

In particular, risk-based testing can help in

- providing evidence on the functional correctness of countermeasures,

- providing evidence on the absence of known vulnerabilities, and

- discovering unknown vulnerabilities,

- optimizing risk analysis by identifying new risk factors and reassessing the risk values.

The CORAS language [7] integrates different tree-based approaches for risk modelling. It is a graphbased modelling approach that emphasizes the modelling of threat scenarios and provides formalisms to annotate the threat scenarios with probability values and formalisms to reason with these annotations.

Figure 2 shows a simple CORAS risk model that depicts a threat scenario for an unauthorized database access. The CORAS language allows to relate threat scenarios to adversaries (so called threats, e.g. "'hacker"" or "'script kiddy" in Figure 2) and to potential vulnerabilities. A vulnerability is denoted by the unlocked padlock (see e.g. "'SQL injection"'). Last but not least the threat scenario is related to unwanted incidents, e.g. "Database entries are modified by unauthorized people". This modification harms the asset "database contents" (see "brown moneybag"' in Figure 2) and can therefore negatively influence the asset "'revenue" (see "white moneybag"). The treatment "use of prepared statements" instead of strings containing SQL queries is depicted with the green pliers in that figure. Except the 


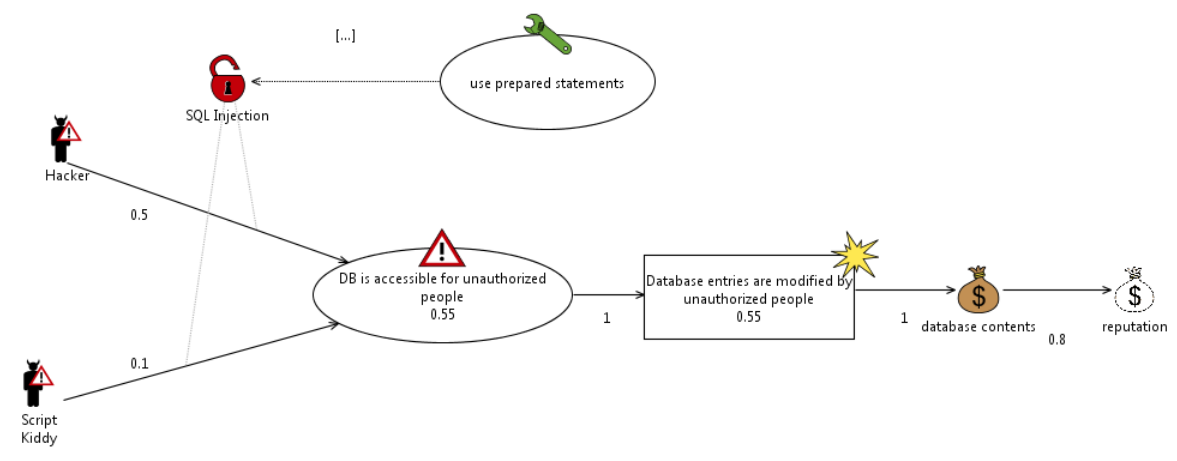

Figure 2: CORAS treatment diagram

threats, the vulnerability, the assets and the treatment, all elements have annotations that denote the probability or frequency of the transition or the incidence of for instance a threat scenario.

Such risk models can be used in different ways to support testing. The goal of risk-based test identification is to improve the test design such that high-risk areas of the SUT are covered and that at the same time test resources are optimally used by focusing on highest risks first.

In risk-based test identification, for individual risk factors test objectives are developed. We consider a test objective to be foremost an informal specification that defines which aspect of a certain system, functionality, or protocol etc. should be tested. Similar to requirements in requirements engineering, test objectives constitute test requirements that can be refined and decomposed during the test development. In the following we describe the relationship between test objectives and the elements used in the risk analysis. Quantifications of the related risks can be used to weight the test objectives.

Test objectives for

- an unwanted incident describe which test methods can be applied to initiate and detect an unwanted incident and to characterize its consequences.

- threat scenarios describe which test methods can be applied to initiate a threat scenario and to characterize its consequences.

- vulnerabilities describe which test methods can be applied to elicit a vulnerability.

- treatment scenarios describe which test methods can be applied to characterize the maturity and effectiveness of a treatment scenario.

Another way of using risk models in testing is risk-based test selection. It is used to find an optimal set of test cases along certain selection strategy. The selection strategy takes into account available test resources and optimizes the selected tests with respect to the chosen coverage criteria. In functional testing coverage is often described by the coverage of requirements or by the coverage of system or test model elements such as states, transitions, or decisions. In risk-based testing, we aim at the coverage of system risks. The criteria are designed by taking the risk values from the risk assessment to set priorities for the test generation or to order the test execution in a test run. The test selection can be either accomplished on existing test cases to select tests for test run or during test generation to enable the directed, goal-oriented generation of tests.

Last but not least, security testing supports risk control. Risk control deals with the revision of risk assessment results by correcting assumptions on probabilities, consequences or the maturity of treatments scenarios or deals with the completion of risk analysis result by integrating vulnerabilities and thus 
potentially threats, threat scenarios and unwanted incidents. The test results can reflect and verify the assumptions that have been made during risk analysis. The test results can be used to adjust risk analysis results by introducing new or revised vulnerabilities or revised risk estimations on basis of the defects being found. Test results, test coverage information and a revised or affirmed risk assessment can provide solid arguments for the security level of a system as test results relate to the

- risks, which are addressed and covered by related test cases.

- treatment or threat scenarios, which are to be checked by the test cases.

- assets, whose related risks are checked by corresponding test cases.

- vulnerabilities, which have been identified and located related test cases.

\subsubsection{Model-Based Fuzzing}

While the origin of fuzzing is based on a complete randomized approach [15], block-based and modelbased fuzzers use their knowledge about the message structure to systematically generate messages containing invalid data among valid data [19].

Systematic approaches are often more successful because the message structure is preserved and thus the likelihood increases that the generated message is accepted by the SUT. Using fuzz testing principles not only for test data generation but also for test behaviour generation complements the traditional fuzz testing approaches. Behaviour fuzzing does not only reflect the generation of atypical messages but also changes the typical appearance and order of messages. For example a valid and approved sequence of messages can be turned into an atypical and unknown sequence by rearranging messages, repeating and dropping them or just by changing the type of message.

Behaviour fuzzing aims at finding flaws in design and vulnerabilities in systems that are not simply revealed by applying invalid input data. It focuses on misuse on a higher level of functionality. For example, a security requirement defines that a download may only be started after successful authentication. In a vulnerable system the download can be started additionally without any authentication. Such fault can be detected using typical input data but atypical behaviour, e.g. by simply omitting the authentication.

DIAMONDS develops model-based fuzzing approaches that use e.g. fuzzing operators on scenario models which are specified by sequence diagrams. In the following, a simplified example from the banking domain is used to illustrate how fuzzing operators are applied to sequence diagrams. For ease of understanding most parameters are omitted.

The sequence diagram in Figure 3 describes how a bank customer can perform a transfer order. The customers can either order a national or an international transfer (message 1). Afterwards the customer sends the name of the recipient of the transfer order, the amount to be transferred (message 2) as well as recipient's national bank account information (message 3 ) in case of a national transfer order or recipient's international bank account information (message 4) in case of an international transfer order. The transfer order must be authorized by the customer sending a valid transaction number TAN (message 5). If the customer accidentally sent an invalid TAN e. g. by mistyping it, he can try to enter a valid TAN up to two times again (message 7, combined fragment loop).

Applying fuzzing operators to the diagram, messages can be moved, removed, repeated, inserted or the type of a message can be changed to obtain an invalid sequence. Fuzzing operators perform a mutation on the diagram resulting in an invalid sequence in comparison with the original. One fuzzing operators performs only one mutation of a sequence diagram. For instance, a fuzzing operator can move 


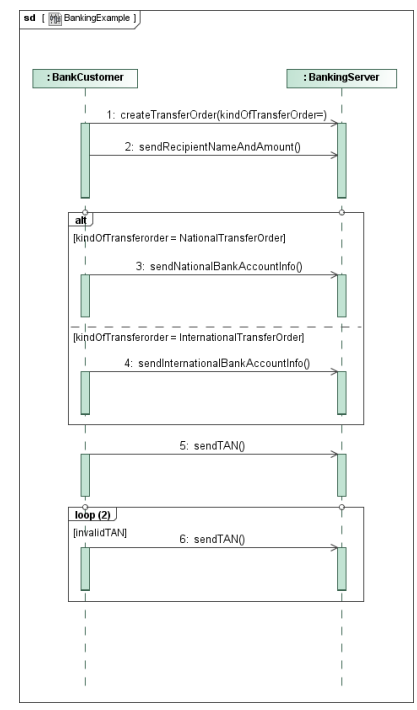

Figure 3: Transfer Order Sequence

message 5 after message 2. Another fuzzing operator can generate an invalid sequence of messages by negating interaction constraints of interaction operands. By negating the interaction constraint of the loop combined fragment, the sequences generated from the resulting sequence diagram contain at least two valid transaction numbers sent to the banking server (three if the second given TAN is valid). Figure 4 shows the results of the fuzzing operators from above.
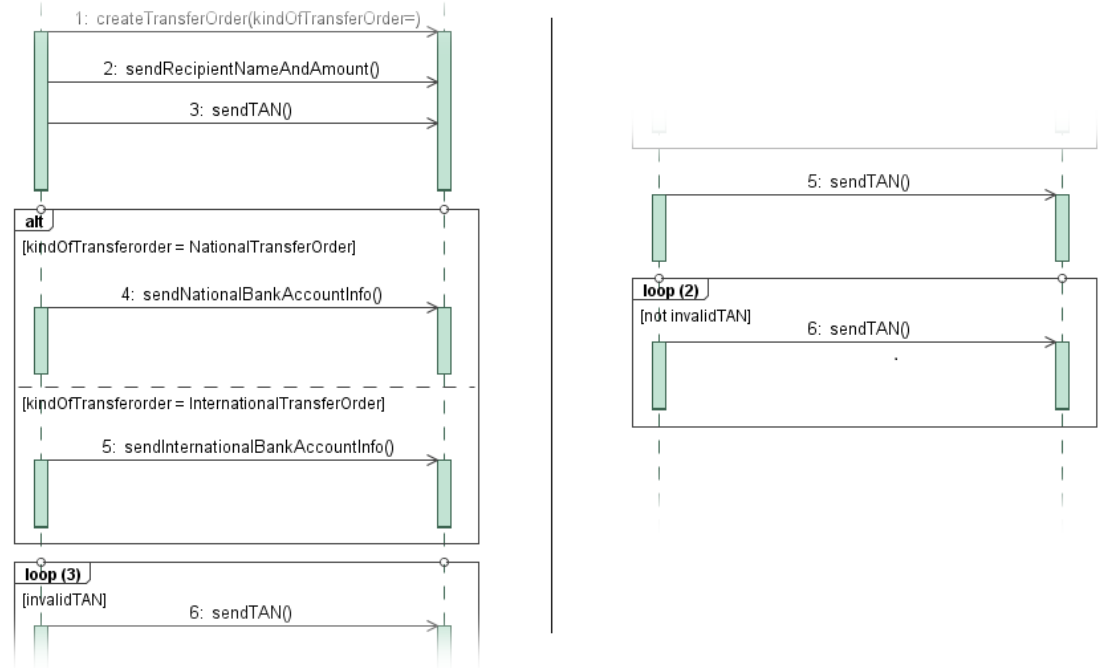

Figure 4: Fuzzed Transfer Order Sequence

Performing the above mentioned fuzzing operators leads to different sequence diagrams that are the basis for further test case generation. However, the main idea of fuzz testing approaches in general is the ability to automatically generate a large number of test cases. This is achieved by applying not only one fuzzing operator to a sequence diagram, but a set of fuzzing operators multiple times, e.g. 
by applying a single fuzzing operator to several model elements of a sequence diagram or by applying several, possibly different fuzzing operators, one after another. The combination of fuzzing operators permits the generation of a large number of test cases.

\section{Summary}

Model-based security testing (MBST) is a relatively new field and especially dedicated to the systematic and efficient specification and documentation of security test objectives, security test cases and test suites, as well as to their automated or semi-automated generation. This paper provides an initial survey on model-based security testing by analyzing related work, discussing models that can be used for modelbased security testing, and by outlining two main approaches that are being developed in the European ITEA2 project DIAMONDS by industrial and research partners from 6 countries:

- Risk-based security testing

- Model-based fuzzing

Details of risk-based security testing and model-based fuzzing are given in the DIAMONDS deliverables. While DIAMONDS is an ongoing project that is at the half of the project duration having reached 2 of 4 milestone, the methods are still under development and the analysis of the gains and the pros and cons of the methods is still to be done. However, initial versions of the methods have already been applied in selected case studies that demonstrated the potentials of the described approaches.

\section{References}

[1] Paul Baker, Zhen Ru Dai, Jens Grabowski, ystein Haugen, Ina Schieferdecker \& Clay Williams (2007): Model-Driven Testing: Using the UML Testing Profile, 1 edition. Springer, Berlin. Available at http: //dx.doi.org/10.1007/978-3-540-72563-3.

[2] David Basin, Jürgen Doser \& Torsten Lodderstedt (2006): Model driven security: From UML models to access control infrastructures. ACM Trans. Softw. Eng. Methodol. 15, pp. 39-91. Available at http: //doi.acm.org/10.1145/1125808.1125810

[3] Mark Blackburn, Robert Busser \& Aaron Nauman (2002): Model-based approach to security test automation. In: International Software Quality Week.

[4] Paul Gerrard \& Neil Thompson (2002): Risk Based E-Business Testing. Artech House, Inc., Norwood, MA, USA.

[5] F. Y. Gu Tian-yang, Shi Yin-sheng \& Yuan (2010): Research on Software Security. Testing World Academy of Science Engineering and Technology 692010.

[6] Matthias Güdemann, Frank Ortmeier \& Wolfgang Reif (2007): Using Deductive Cause Consequence Analysis (DCCA) with SCADE. In: Proceedings of SAFECOMP 2007, Springer LNCS 4680.

[7] Ida Hogganvik (2007): A Graphical Approach to Security Risk Analysis. Ph.D. thesis, Oslo : University of Oslo, Department of Informatics.

[8] Jan Jürjens (2002): UMLsec: Extending UML for Secure Systems Development. In Jean-Marc Jézéquel, Heinrich Hussmann \& Stephen Cook, editors: The Unified Modeling Language, Lecture Notes in Computer Science 2460, Springer Berlin / Heidelberg, pp. 1-9. Available at http://dx.doi.org/10.1007/ 3-540-45800-X_32

[9] Jan Jürjens (2005): Secure Systems Development with UML. Springer. Available at http://dx.doi.org/ $10.1007 / \mathrm{b} 137706$ 
[10] Jan Jürjens (2008): Model-based Security Testing Using UMLsec. Electron. Notes Theor. Comput. Sci. 220, pp. 93-104. Available at http://dl.acm.org/citation.cfm?id=1467086.1467133

[11] Jan Jürjens \& Guido Wimmel (2001): Specification-Based Testing of Firewalls. In Dines Bjørner, Manfred Broy \& Alexandre V. Zamulin, editors: Ershov Memorial Conference, Lecture Notes in Computer Science 2244, Springer, pp. 308-316. Available at http://dx.doi.org/10.1007/3-540-45575-2_31.

[12] Rauli Kaksonen (2001): A functional method for assessing protocol implementation security. VTT Publications 448, VTT Technical Research Center of Finland.

[13] M. S. Lund, B. Solhaug \& K. Stlen (2011): Model-Driven Risk Analysis. The CORAS Approach. ISBN: 978-3-642-12322-1, Springer.

[14] Sjouke Mauw \& Martijn Oostdijk (2005): Foundations of Attack Trees. In: International Conference on Information Security and Cryptology ICISC 2005. LNCS 3935, Springer, pp. 186-198.

[15] Barton P. Miller, Lars Fredriksen \& Bryan So (1990): An Empirical Study of the Reliability of UNIX Utilities. In: In Proceedings of the Workshop of Parallel and Distributed Debugging, Academic Medicine, pp. pages ix-xxi,.

[16] Tejeddine Mouelhi, Franck Fleurey, Benoit Baudry \& Yves Le Traon (2008): A Model-Based Framework for Security Policy Specification, Deployment and Testing. In Krzysztof Czarnecki, Ileana Ober, Jean-Michel Bruel, Axel Uhl \& Markus Völter, editors: MoDELS, Lecture Notes in Computer Science 5301, Springer, pp. 537-552. Available at http://dx.doi.org/10.1007/978-3-540-87875-9_38

[17] D.S. Nielsen (1971): The Cause/Consequence Diagram Method as a Basis for Quantitative Accident Analysis. Technical Report RISO-M-1374, Danish Atomic Energy Commission.

[18] K.A. Reay \& University of Loughborough (2002): Efficient fault tree analysis using binary decision diagrams/. University of Loughborough. Available at http://books.google.de/books?id=_0SFGwAACAAJ

[19] A. Takanen, J. DeMott \& C. Miller (2008): Fuzzing for software security testing and quality assurance. Artech House information security and privacy series, Artech House. Available at http://books google . de/books?id=tMuAc_y9dFYC

[20] W E Vesely, F F Goldberg, N H Roberts \& D F Haasl (1981): Fault Tree Handbook. Office (NUREG-0492), p. 209. Available at http://www.stormingmedia.us/37/3794/A379453.pdf.

[21] Linzhang Wang, Eric Wong \& Dianxiang Xu (2007): A Threat Model Driven Approach for Security Testing. In: Proceedings of the Third International Workshop on Software Engineering for Secure Systems, SESS '07, IEEE Computer Society, Washington, DC, USA, pp. 10-. Available at http://dx.doi.org/10. 1109/SESS. 2007.2.

[22] Martin Weiglhofer, Bernhard K. Aichernig \& Franz Wotawa (2009): Fault-Based Conformance Testing in Practice. Int. J. Software and Informatics 3(2-3), pp. 375-411. Available at http://www .ijsi.org/IJSI/ $\mathrm{ch} /$ reader/view_abstract. aspx?file_no $=375\{\&\}$ flag $=1$.

[23] Guido Wimmel \& Jan Jürjens (2002): Specification-Based Test Generation for Security-Critical Systems Using Mutations. In: Proceedings of the 4th International Conference on Formal Engineering Methods: Formal Methods and Software Engineering, ICFEM '02, Springer-Verlag, London, UK, UK, pp. 471-482. Available at http://dl.acm.org/citation. cfm?id=646272.685812 